Acta vet. scand. $1973,14,761-763$.

Brief Communication

\title{
DISTRIBUTION OF SOME MINERAL COMPONENTS \\ IN THE FOETAL FLUID \\ OF THE GOAT AT DIFFERENT STAGES OF PREGNANCY
}

Amniotic and allantoic fluid samples were collected at different stages of pregnancy in the goat (Lyngset 1971). After collection the samples were stored in a frozen state until processed.

The fluid samples were analysed with regard to the minerals $\mathrm{Ca}, \mathrm{P}, \mathrm{Mg}, \mathrm{Na}, \mathrm{K}, \mathrm{Fe}, \mathrm{Zn}$ and $\mathrm{Cu}$. The metals were determined by atomic absorption analysis, after wet ashing of the fluids with sulphuric and nitric acid (5 g fluid and $0.25 \mathrm{ml} \mathrm{H}_{2} \mathrm{SO}_{4}$ ). Phosphorus was determined spectrophotometrically as phosphoammoniummolybdate after the same ashing procedure.

Parallel with this investigation analyses of the oestrogenic content in the same foetal fluid samples were undertaken (Lyngset \& Lunaas 1972).

The results of the mineral analyses are given in Table 1. As the amount of fluid varied, there were not sufficient quantities available for analyses at all stages, leaving these stages with no results in the table.

The contents were higher in the allantoic fluid than in the amniotic fluid for all the minerals under survey with the exception of $\mathrm{Na}$, which showed a reverse pattern. This has earlier been stated in sheep with respect to $\mathrm{Na}, \mathrm{K}, \mathrm{Ca}, \mathrm{Mg}$ and $\mathrm{P}$ (McDougall 1949).

For the macrominerals ( $\mathrm{Ca}, \mathrm{Mg}, \mathrm{K}, \mathrm{Na}$ and $\mathrm{P}$ ) the differences between the allantoic and amniotic fluid were statistically significant, as proved by analysis of variance. An equal significance could not be proved for the microminerals.

There is a relatively great fluctuation in the mineral content throughout the whole gestational period in both fluid compartments, but for the content of $\mathrm{Ca}, \mathrm{Mg}$ and $\mathrm{K}$ there seems to be a marked increase in the allantoic fluid at approx. the 90th day of 
T a b le 1. Foetal fluid contents of the elements $\mathrm{Ca}, \mathrm{Mg}, \mathrm{K}, \mathrm{Na}, \mathrm{Fe}, \mathrm{Zn}, \mathrm{Cu}$ and $P$ in the goat.

\begin{tabular}{|c|c|c|c|c|c|c|c|c|}
\hline \multirow[t]{2}{*}{$\begin{array}{c}\text { Day of } \\
\text { pregnancy }\end{array}$} & \multicolumn{2}{|c|}{$\begin{array}{c}\text { Ca } \\
\text { p.p.m. }(w / w)\end{array}$} & \multicolumn{2}{|c|}{$\begin{array}{c}\mathbf{M g} \\
\text { p.p.m. }(\mathbf{w} / \mathbf{w})\end{array}$} & \multicolumn{2}{|c|}{$\begin{array}{c}\mathbf{K} \\
\text { p.p.m. }(\mathbf{w} / \mathbf{w})\end{array}$} & \multicolumn{2}{|c|}{$\begin{array}{c}\text { Na } \\
\text { p.p.m. }(w / w)\end{array}$} \\
\hline & all. & amn. & all. & amn. & all. & amn. & all. & amn. \\
\hline 26 & 25 & - & 8.4 & - & 240 & - & 1600 & - \\
\hline 45 & 72 & 29 & 11 & 7.9 & 180 & 590 & 3300 & 3000 \\
\hline 60 & 67 & 46 & 16 & 13 & 190 & 540 & 780 & 3300 \\
\hline 60 & 13 & 24 & 32 & 8.8 & 360 & 550 & 2200 & 3800 \\
\hline 75 & 32 & 20 & 23 & 9.5 & 320 & 420 & 1200 & 3000 \\
\hline 75 & 38 & 23 & 32 & 9.0 & 240 & 290 & 2400 & 2700 \\
\hline 90 & 160 & 22 & 100 & 6.7 & 330 & 150 & 750 & 1200 \\
\hline 90 & 320 & 18 & 290 & 4.8 & 1300 & 150 & 630 & 1100 \\
\hline 105 & 180 & 24 & 160 & 7.2 & 1400 & 120 & 920 & 1000 \\
\hline 105 & - & 35 & - & 25 & - & 310 & - & 2300 \\
\hline 105 & 160 & 99 & 260 & 87 & 180 & 220 & 670 & 2800 \\
\hline 120 & 240 & 21 & 240 & 6.4 & 2200 & 160 & 770 & 730 \\
\hline 121 & 45 & 40 & 150 & 18 & 2600 & 320 & 1500 & 3400 \\
\hline 130 & 150 & 100 & 180 & 29 & 900 & 470 & 160 & 1900 \\
\hline 140 & 47 & 31 & 160 & 30 & 950 & 270 & 200 & 1700 \\
\hline 140 & 100 & 48 & 560 & 110 & 2300 & 600 & 150 & 1700 \\
\hline 145 & 140 & 24 & 170 & 9.4 & 1600 & 200 & 100 & 1300 \\
\hline \multirow[t]{2}{*}{ Mean } & 112 & 38 & 150 & 23.9 & 956 & 335 & 1083 & 2183 \\
\hline & \multicolumn{2}{|c|}{$\mathrm{P}<0.001$} & \multicolumn{2}{|c|}{$0.001<\mathrm{P}<0.01$} & \multicolumn{2}{|c|}{$0.001<\mathrm{P}<0.01$} & \multicolumn{2}{|c|}{$0.001<\mathrm{P}<0.01$} \\
\hline
\end{tabular}

\begin{tabular}{|c|c|c|c|c|c|c|c|c|}
\hline \multirow[t]{2}{*}{$\begin{array}{l}\text { Day of } \\
\text { pregnancy }\end{array}$} & \multicolumn{2}{|c|}{$\begin{array}{c}F e \\
\text { p.p.m. }(\mathbf{w} / \mathbf{w})\end{array}$} & \multicolumn{2}{|c|}{$\begin{array}{c}\mathrm{Zn} \\
\text { p.p.m. }(\mathbf{w} / \mathbf{w}) \\
\end{array}$} & \multicolumn{2}{|c|}{$\begin{array}{c}\mathrm{Cu} \\
\text { p.p.m. }(\mathbf{w} / \mathbf{w})\end{array}$} & \multicolumn{2}{|c|}{$\begin{array}{c}\mathbf{P} \\
\text { p.p.m. }(\mathbf{w} / \mathbf{w})\end{array}$} \\
\hline & all. & amn. & all. & amn. & ail. & amn. & all. & amn. \\
\hline 26 & 1.4 & - & 0.25 & - & 0.13 & - & - & - \\
\hline 45 & 7.7 & 0.28 & 0.28 & 0.33 & 0.17 & 0.13 & 21 & 22 \\
\hline 60 & 6.1 & $<0.05$ & 0.33 & 0.43 & 0.20 & 0.16 & 19 & 28 \\
\hline 60 & 3.6 & $<0.05$ & 0.40 & - & 0.30 & 0.08 & 67 & 20 \\
\hline 75 & 6.3 & 0.45 & 0.49 & 0.24 & 0.28 & 0.10 & 42 & 14 \\
\hline 75 & 0.25 & 0.85 & 0.05 & 0.37 & 0.13 & 0.07 & 18 & 26 \\
\hline 90 & 7.9 & 0.10 & 0.30 & - & $<0.05$ & $<0.05$ & 40 & 12 \\
\hline 90 & 0.38 & $<0.05$ & 0.23 & 0.03 & $<0.05$ & $<0.05$ & 89 & 3.7 \\
\hline 105 & 11 & 1.1 & 0.30 & 0.35 & 0.20 & 0.16 & 27 & 22 \\
\hline 105 & - & - & - & 0.18 & - & 0.10 & - & 11 \\
\hline 105 & 7.4 & 9.7 & 0.33 & 0.31 & 0.13 & 0.33 & 17 & 22 \\
\hline 120 & 2.7 & 0.78 & 0.34 & 0.12 & 0.13 & - & 71 & 21 \\
\hline 121 & 0.45 & 3.5 & 0.50 & 0.36 & 0.07 & 0.13 & 25 & 24 \\
\hline 130 & 4.6 & 6.1 & 0.20 & 0.61 & 0.12 & 0.23 & 26 & 37 \\
\hline 140 & $<0.05$ & 1.1 & 0.63 & 0.05 & $<0.05$ & $<0.05$ & 15 & 6.1 \\
\hline 140 & 0.30 & 0.95 & 0.16 & 0.15 & 0.07 & - & 38 & 32 \\
\hline 145 & $<0.05$ & 1.4 & 0.04 & 0.05 & 0.13 & $<0.05$ & 34 & 66 \\
\hline \multirow[t]{2}{*}{ Mean } & 3.76 & 1.76 & 0.30 & 0.26 & 0.14 & 0.11 & 36.6 & 22.9 \\
\hline & \multicolumn{2}{|c|}{$0.05<\mathrm{P}<0.2$} & \multicolumn{2}{|c|}{ Not signif. } & \multicolumn{2}{|c|}{ Not. signif. } & \multicolumn{2}{|c|}{$0.01<\mathrm{P}<0.05$} \\
\hline
\end{tabular}


gestation. For $\mathrm{Na}$ there is a corresponding decrease in the content at the same stage. No such pattern is observed in the amniotic fluid.

As stated by Mellor \& Slater (1971) the sampling technique could be of significance, but is considered to be negligible in the present work.

Gustav N. Havre and Olav Lyngset

The Department of Biochemistry, and

The Division of Postgraduate and Continuing Education,

Veterinary College of Norway, Oslo.

\section{REFERENCES}

Lyngset, O.: Studies on reproduction in the goat. VII. Pregnancy and the development of the foetus and the foetal accessories of the goat. Acta vet. scand. $1971,12,185-201$.

Lyngset, $O$. \& T. Lunaas: Amniotic and allantoic fluid levels of oestrogens at different stages of development in the goat. Acta vet. scand. 1972, 13, 269-271.

McDougall, E. L.: The composition of foetal fluids of sheep at different stages of gestation. Biochem. J. 1949, 45, 397-400.

Mellor, D. J. \& J. S. Slater: Daily changes in amniotic and allantoic fluid during the last three months of pregnancy in conscious, unstressed ewes, with catheters in their foetal fluid sacs. J. Physiol. (Lond.) 1971, 217, 573-604.

(Received November 1, 1973).

Reprints may be requested from: G. N. Havre, Veterinary College of Norway, PO Box 8146, Oslo Dep., Oslo 1, Norway. 